\title{
Investigation of using RFID for cure monitoring of glass fiber-reinforced plastics
}

\author{
Marius Veigt ${ }^{1}$ (1) Elisabeth Hardi $^{2} \cdot$ Michael Koerdt $^{2} \cdot$ Axel S. Herrmann $^{2,3} \cdot$ Michael Freitag $^{1,3}$
}

Received: 11 February 2020 / Accepted: 10 July 2020 / Published online: 16 July 2020

(c) The Author(s) 2020

\begin{abstract}
Fiber composite components play an important role in the turnaround in energy policy as well as in stopping global warming. Therefore, it is essential to improve the manufacturing efficiency of these components. RFID technology is spreading to digitize and organize processes in production and logistics more efficiently. Since cure monitoring is a crucial factor in the manufacturing of composite components, the question arises whether the RFID technology is applicable for cure monitoring. This paper presents two methods of how an into glass fiber-reinforced plastics integrated RFID transponder could monitor the curing. Following the assumption that the change in permittivity of the glass fiber-reinforced plastic during curing influence the RFID signal, experiments in a measuring chamber (low-interference environment) were conducted. It was investigated whether the optimal response frequency of the integrated RFID transponder changes and whether the received signal strength indicator (RSSI) changes at a specific frequency during curing. As a reference method, the dielectric analysis as a well-known method for cure monitoring was used and compared with the RFID measurements. The results indicate that the optimal response frequency remains constant but the RSSI increases and possess a very high linear correlation with the measurement of the dielectric analysis in a low-interference environment. Consequently, the RFID technology is applicable to monitor the curing of glass fiber-reinforced plastics by measuring the RSSI in a low-interference environment.
\end{abstract}

Keywords RFID · UHF · Glass fiber-reinforced plastics · Cure monitoring

\section{Introduction}

In the past years, a continuous increase of the production volume of fiber-reinforced plastics (FRP) can be observed [1]. Reasons for the increase are that these materials play a major role in the turnaround in energy policy, as they are an essential part of wind turbines, and in stopping global warming. The pressure to reduce $\mathrm{CO}_{2}$ emissions is leading to increased efforts to make aircrafts, cars, trucks, and the like, more lightweight. FRP offer advantageous material properties for lightweight construction $[1,2]$.

Marius Veigt

vei@biba.uni-bremen.de

1 BIBA-Bremer Institut für Produktion und Logistik GmbH, Hochschulring 20, 28359 Bremen, Germany

2 Faserinstitut Bremen e. V., Am Biologischen Garten 2, 28359 Bremen, Germany

3 Faculty of Production Engineering, University of Bremen, Bibliothekstr. 1, 28359 Bremen, Germany
In order to achieve the advantageous material properties, it is essential to cure the components in a targeted manner. Cure monitoring can improve both the quality and efficiency of the curing process of FRP [3]. Thus, there are many current research activities to enhance the conventional cure monitoring methods, like the dielectric analysis [4], the differential scanning calorimetry [5], the dynamic mechanical analysis [6] and ultrasound monitoring [7, 8]. However, these conventional methods are criticized because they require additional equipment, processes, and expertise generating additional costs without bringing any further benefit (beside the cure monitoring) in the product life cycle [9].

Konstantopoulos et al. observed a trend towards "sensing materials" [10], such as fiber Bragg sensors, which can be used both for cure monitoring [11] and for structure health monitoring [10]. However, these optical fibers are fragile and susceptible to mechanical stress [12]. Hence, there is a high risk of damaging the sensors, which leads to increased costs and inefficiency [10]. 
Consequently, besides the enhancement of conventional cure monitoring methods, new methods for cure monitoring are being pursued [13].

At the same time, a digitalization of the manufacturing process is demanded in order to reduce manufacturing costs of FRP [14]. In this context, radio frequency identification (RFID) technology comes into focus. Simulation studies indicate that the integration of RFID transponders into FRP components provides the basis for reducing throughput times, inventories, and rejects in FRP manufacturing as well as improving the due date adherence $[15,16]$. Moreover, RFID enables an autonomous production [17]. In addition, RFID offers the possibility to document the manufacturing and usage processes locally [15]. The resulting component history can be used for maintenance and component authentication [18].

This contribution examines the feasibility of using RFID for cure monitoring of FRP. Two measurement methods have been investigated in an experiment. The results have been compared to a reference method and discussed. The next section presents the state of the art and identifies the research gap. The third section introduces the measurement background and the fourth section describes the experimental design. It is followed by the results and a discussion in section five. In the sixth section, the contribution concludes with a summary and an outlook on further research.

\section{Related work and research gap}

Zettler et al. and Gray demonstrated RFID transponders integrated in FRP could still be read. Both authors investigated the effect of RFID integration on the final mechanical performance of the FRP $[19,20]$. Gray showed how to predict the influence of the RFID transponder on the mechanical properties by FEM simulations and, thus, how to determine a suitable transponder position [20].

Bernhard et al. conducted extensive tests to determine the influence of FRP on the reading range of RFID systems. Their results showed that carbon fiber-reinforced plastics strongly attenuate the RFID signal [21, 22]. However, glass fiber-reinforced plastics (GFRP) barely influence the reading range of low frequency and high frequency systems. The reading range of ultra high frequency (UHF) systems decreases due to GFRP [21]. Furthermore, there was a shift in the best reading frequency. While the RFID transponders achieved the maximum reading range in free space at $868 \mathrm{MHz}$, the laminated transponders reached the maximum reading range at approx. $800 \mathrm{MHz}$. Considering $868 \mathrm{MHz}$ the theoretical read range dropped from 8 to $5 \mathrm{~m}$ [21].

Hardi et al. used this effect to monitor the resin flow during a vacuum infusion of GFRP using an integrated UHF RFID transponder. In contrast to Bernhard et al., Hardi et al. measure the received signal strength indicator (RSSI). With experiments, they proved that this value drops significantly as soon as the resin flow reaches the integrated RFID transponder [23].

For the first time, Veigt et al. demonstrated experimentally that the received signal strength indication (RSSI) of an RFID transponder integrated into FRP increases synchronously with the curing process. This was proofed by comparing the RSSI measurements with a reference measurement of the dielectric analysis (DEA). In summary, the result shows that cure monitoring of GFRP and aramid FRP is possible by using UHF RFID transponder [24, 25]. During curing, the dielectric properties of the GFRP change. This change affects the strength of the response signal of an integrated RFID transponder. However, reflections and other radio waves disturb the RSSI measurement. These interferences can be balanced by averaging the RSSI of several transponders [26], but there is still potential to enhance the measurement to make it more precise.

Groth et al. developed wireless and chipless sensors, which can be integrated into plastics and can be used at $25 \mathrm{GHz}$ [13] or at $46 \mathrm{GHz}$ [27]. These sensors shift their best response frequency during the curing process resulting from the dielectric permittivity change of the plastic. The shift of the best response frequency correlates with the curing process, which was proofed by comparing the shift with the measurement result of a differential scanning calorimetry.

Measuring the frequency shift has the advantage that signal reflections do not affect the measuring, unlike the RSSI. However, the use of UHF RFID transponders has the advantage that these standardized transponders are both inexpensive and offer further advantages in product life. For instance, they can be used in logistics and maintenance processes.

An open research gap is whether a frequency shift correlating with the curing process of GFRP occurs within the UHF range. This frequency shift could make the measuring of the cure monitoring less easily disturbed by interferences. Another open research gap is which impact the interference has on the RSSI measurement. This contribution examines these two gaps.

\section{Measurement background}

\subsection{Radio frequency identification}

An RFID system consists of a couple of components. The transponders are attached to objects to make them identifiable. An RFID reader (and writer) consists of a highfrequency module (transmitter and receiver), a control unit and one or more antennas. The RFID reader supplies the transponders with energy via air interface. A minimum field 
strength $E$ is required to activate the transponder and supply it with sufficient energy for data transmission. Equation (1) expresses the minimum field strength $\mathrm{E}_{\min }$ [28]:

$E_{\text {min }}=\sqrt{\frac{4 \pi \cdot \sqrt{\mu * \varepsilon} \cdot P_{e-\min }}{\lambda^{2} \cdot G}}$,

where $\mu$ is the magnetic permeability and $\varepsilon$ the electric permittivity of the transmission medium, $\mathrm{P}_{\mathrm{e}-\mathrm{min}}$ is the minimum required input power, $\lambda$ is the wavelength, which depends on the frequency, and $\mathrm{G}$ is the transponder antenna gain. Further, the arriving field strength $\mathrm{E}$ depends on the transmission power of the reader $\mathrm{P}_{\text {EIRP }}$, the magnetic permeability $\mu$ and the electric permittivity $\varepsilon$ of the transmission medium as well as the distance $r$ between the reader antenna and the transponder antenna, see Eq. (2) [28]:

$E=\sqrt{\frac{P_{E I R P} \times \sqrt{\mu \times \varepsilon}}{4 \pi r^{2}}}$.

The strength of a received signal, also called backscatter signal, can be measured utilizing the RSSI. It is only an indicator because interferences, such as other radio waves and reflections, affecting the measurement.

RFID systems exist in many different variants. A significant distinguishing feature is the operating frequency. For low frequency systems, the used frequencies are between 30 and $300 \mathrm{kHz}$, for high frequency systems between 3 and $30 \mathrm{MHz}$, for ultra-high frequency (UHF) systems between $300 \mathrm{MHz}$ and $3 \mathrm{GHz}$, and for microwave above $3 \mathrm{GHz}$. In case of application in production planning and control as well in logistics, the UHF is established. In Europe, the frequency range for UHF application is $865-868 \mathrm{MHz}$ [28].

\subsection{Dielectric analysis (DEA)}

The DEA is a widely accepted method to characterize the polymeric resins during curing. Free ions and dipoles existing within it affect the dielectric behavior of the resin. In the uncured resin, the free ions can move and thus dominate the dielectric response. In the cured resin, ion migration reduces to nearly zero, and the alignment of the dipoles dominates the dielectric response, which is a frequency-dependent phenomenon [29]. Thus, the complex electrical impedance can be used to monitor the curing of thermoset resins or adhesives [30]. The frequency-independent resistivity or direct current resistivity (also called ion viscosity) correlates with the cure state throughout the curing process. Interdigitated electrodes on a substrate in contact with the material under test can be used as a sensor for studying the dielectric properties and for observation of cure state of the material under test.

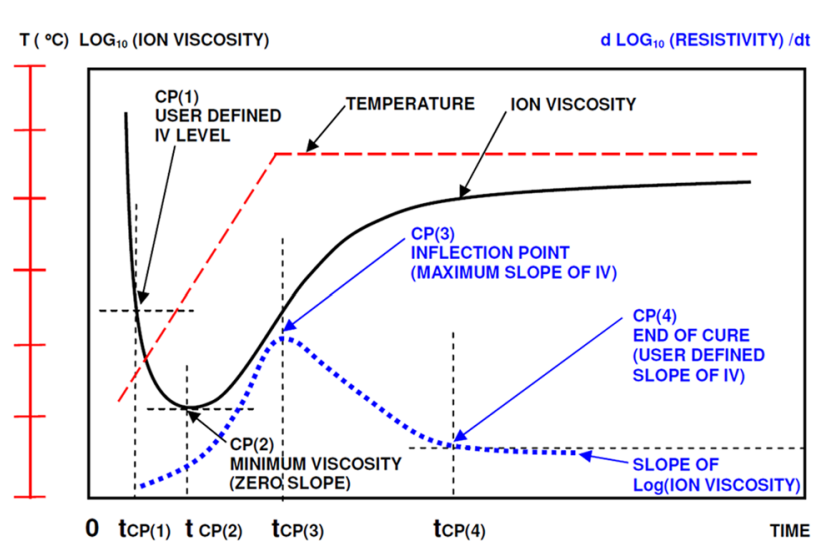

Fig. 1 Measurement of ion viscosity (IV) in FRP by DEA [29]

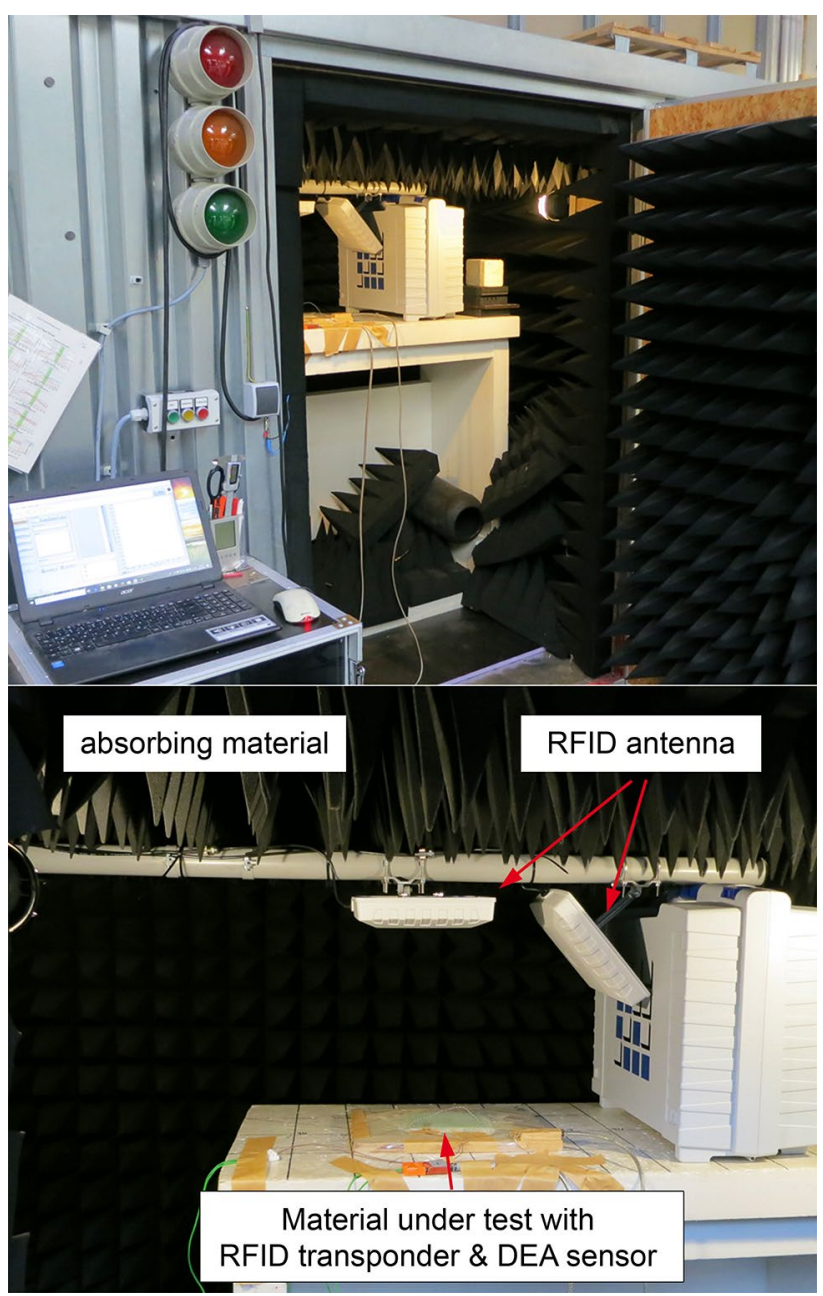

Fig. 2 Measuring chamber equipped with Cisc RFID Xplorer

The change in ion viscosity during the curing process is typically nonlinear, as shown in Fig. 1. Transition phases can be identified by measuring the ion viscosity and by defining 4 points [see $\mathrm{CP}(1)$ to $\mathrm{CP}(4)$ in Fig. 2] [29]: 
1. The decrease in ion viscosity at the beginning indicates the wetting of the sensor with the resin. The user can define ion viscosity level $\mathrm{CP}(1)$ and use it, e.g. to detect a resign flow.

2. The minimum ion viscosity $\mathrm{CP}(2)$ (typically equivalent to the minimum mechanical viscosity) indicates the time at which the accelerated cross-linking reactions dominate the resin.

3. The inflection point $\mathrm{CP}(3)$ indicates the point in time when the cross-linking reactions begin to slow down.

4. The slope of the curve $\mathrm{CP}(4)$ marks the end of the curing process. The decreasing slope of the curve corresponds to a decreasing reaction rate. The ion viscosity increases only slowly and converges to a constant value at the end of curing. It is user-specific which point in time is considered as the end of curing.

\subsection{Bravais-Pearson correlation coefficient}

In order to assess quantitatively the relationship between the RFID measurement and the DEA measurement we require an appropriate metric. Due to both methods measure the change of the dielectric material properties we expect a linear correlation between the measurement series. The Bravais-Pearson correlation coefficient is a suitable metric to measure the linear correlation of two measurement series [31]. It is calculated by:

$r=r_{x y}=\frac{\sum_{i=1}^{n}\left(x_{i}-\bar{x}\right)\left(y_{i}-\bar{y}\right)}{\sqrt{\sum_{i=1}^{n}\left(x_{i}-\bar{x}\right)^{2}\left(y_{i}-\bar{y}\right)^{2}}}$,

where $x$ is one measurement series and $y$ is the other. The value range of the correlation coefficient lies between 1 and -1 , where 1 is a linear correlation with the same direction and -1 is a linear correlation with the opposite direction. For further interpretation of the correlation of measurement series, the following classification applies: $0.5>|\mathrm{r}|$ is considered as weak correlation, $0.8>|\mathrm{r}| \geq 0.5$ as mean correlation and $|\mathrm{r}| \geq 0.8$ as strong correlation [31].

\section{Experimental setup}

The experiment was conducted in a measuring chamber, see Fig. 2. The measuring chamber blocks radio waves from the environment, ensuring that the measurement is almost free of interferences. In addition, the measuring chamber absorbs the reflections of the RFID system. The used RFID system Cisc RFID Xplorer can perform a frequency between $800 \mathrm{MHz}$ and $1000 \mathrm{MHz}$. It has two antennas. The first antenna transmits energy to the transponder. The second antenna receive the transponder signal. Consequently, the experimental setup was designed to be as interference-free and highly precise as possible.

For the experiment, an E-glass fabric with twill K2/2 and a weight of $390 \mathrm{~g} / \mathrm{m}^{2}$, the EPIKOTE resin L20 and the curing agent $\mathrm{H} 530$ were used. The E-glass fabric was split into 16 layers of $160 \times 160 \mathrm{~mm}$ each. These layers were laminated by hand. During this process, the RFID transponder Protag 3 mini (with an Impinj Monza R6 chip) was centrally integrated into the laminate (between layer 8 and 9); see Fig. 3. The Protag 3 mini is an UHF Gen 2-compliant transponders, which is designed for communications at $865-868 \mathrm{MHz}$. The laminate was cured at a constant room temperature of $23{ }^{\circ} \mathrm{C}$ inside the measuring chamber.

In intervals of $5 \mathrm{~min}$, the frequency sweep from 800 to $1000 \mathrm{MHz}$ was performed in $5 \mathrm{MHz}$ steps and the RSSI of the RFID transponder was measured. Simultaneously, the DEA was conducted with a Netzsch Epsilon 230/10 to generate reference values for the cure monitoring. The DEA sensor was integrated at the side of the material under test between layers 8 and 9, see Fig. 3. Matlab 2019b was used to process the measured data.

\section{Results and discussion}

\subsection{Frequency variation}

Since the transponder is designed for communication between 865 and $868 \mathrm{MHz}$, the highest signal strength of

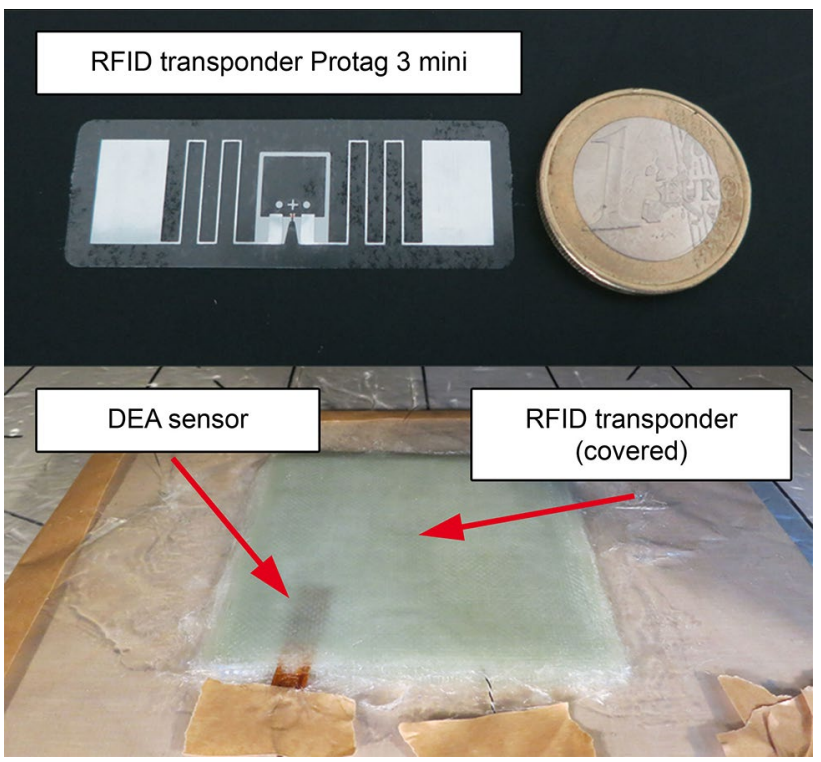

Fig. 3 Used RFID transponder (above) and material under test with integrated sensors (below) 
the transponder was expected at this frequency range when the composite is cured. Further, it was expected that the liquid resin would affect the transponder in such a way that the peak of the RSSI would shift as well as the RSSI would be increase during the curing process. This assumption is based on the physical relationships described in Eqs. 1 and 2. Accordingly, the usable energy by the transponder depends, among others, on the electric permittivity of the transmission medium and the wavelength. Due the changing electric permittivity of the resin, the peak of the RSSI should shift. Following Eq. 2, it was expected that the change of the electric permittivity of the resin would also affect the signal transmission and, thus, the RSSI would increase during the curing process. Figure 4 illustrates this expectation. The red arrow marks the expected shift and increase of the RSSI peak.

Figure 5 presents the result of the frequency variation. For reasons of clarity, the measurement values are connected with lines and only frequency variations at intervals of

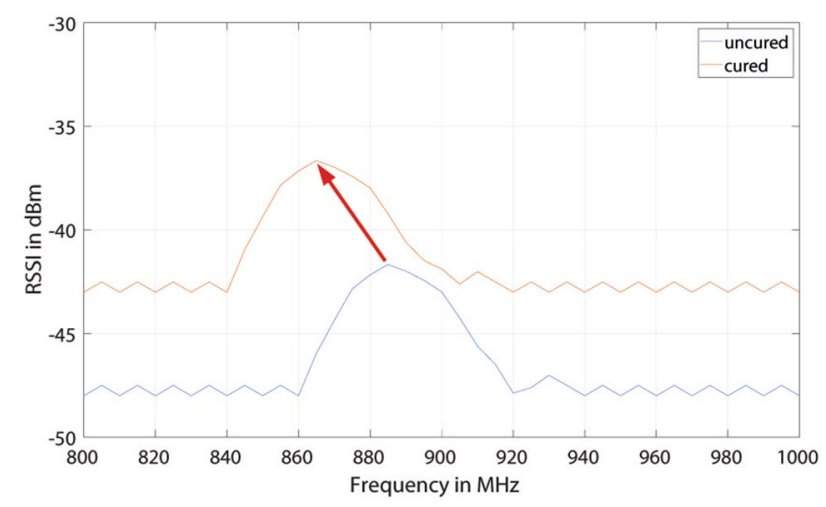

Fig. 4 Expected result of the frequency sweep
$120 \mathrm{~min}$ are displayed. The following findings are obtained from the measurement:

- The RSSI of the integrated RFID transponder is highest at $800 \mathrm{MHz}$, both in cured and uncured state.

- Another peak of the RSSI is around the UHF standard of $865-868 \mathrm{MHz}$, both in cured and uncured state.

- The RSSI increases during the curing process.

Surprisingly, the highest RSSI was measured at $800 \mathrm{MHz}$. However, this is consistent with the result of Bernhard et al. They measured the highest reading range of an in GFRP integrated RFID transponder at $800 \mathrm{MHz}$. In contrast to Bernhard et al., a second peak at approx. $868 \mathrm{MHz}$ was measured. The expected shift of this peak, as observed by Groth et al. in the GHz range, is not detectable in this frequency range with the used RFID transponder. A reason could be the auto-tune function of the used transponder. This function ensures a high performance of the transponder at $868 \mathrm{MHz}$ in different environments.

So far, it can be concluded that no peak shift occurs with a Protag 3 mini between 800 and 1000 MHz. However, possibly this measurement method can work with another RFID transponder without the auto-tune function in this frequency range. Furthermore, a peak shift may also occur with the used transponder in the range below $800 \mathrm{MHz}$ or above $1000 \mathrm{MHz}$.

The question remains where exactly the peak is below $800 \mathrm{MHz}$ and whether this peak shifts. Due to technical restrictions, this question remains open.

\subsection{RSSI measurement}

Considering a specific frequency in Fig. 5, for instance $865 \mathrm{MHz}$, it can be determined that the RSSI increases over
Fig. 5 As a result of the frequency sweeps (displayed every $120 \mathrm{~min}$ ) during curing, the RSSI increases and the peak of the RSSI remains at the same frequency

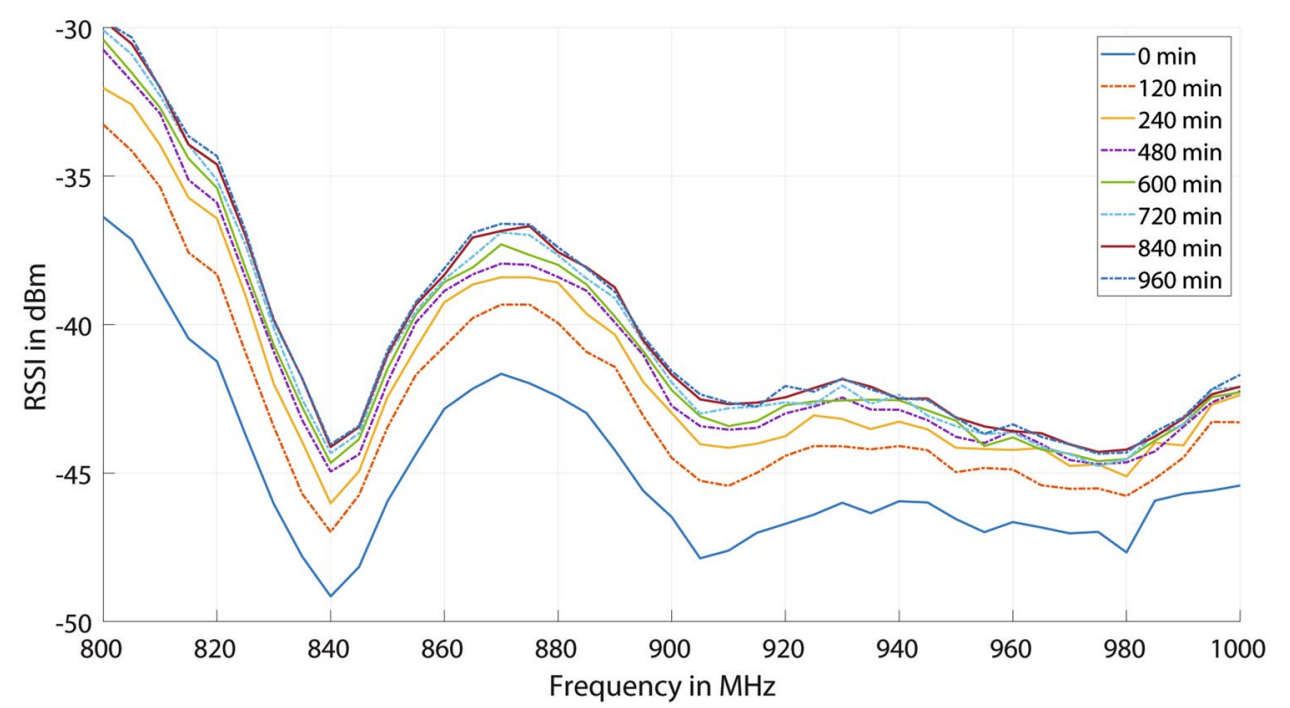


the curing time. Furthermore, the graph shows that this increase is over the first $240 \mathrm{~min}$ higher than during the time afterwards. Figure 6 illustrates the RSSI measurement at $865 \mathrm{MHz}$ over the curing time (black asterisk) and compares this measurement with the ion viscosity measured by dielectric analysis at $1 \mathrm{~Hz}$ (red circle). Furthermore, the blue line displays the core temperature of the material under test. This measurement visualizes the self-heating of the laminate while the curing is starting. The RSSI and the ion viscosity measurements start with a significant slope, which decreases after approx. $300 \mathrm{~min}$ and finally levels out at an almost constant level. In this case, the slope of the ion viscosity is almost zero. However, by remembering the typical curve of ion viscosity illustrated in Fig. 1, it becomes clear that the ion viscosity can also level out with a low slope like the RFID measurement in this case. As the laminate heats up during curing and cools down accordingly at this late stage of curing, the slight increase of the RSSI may indicate that the RSSI measurement is influenced by temperature. As this is important for increasing the precision of the RSSI measurement (especially for curing in an oven or an autoclave), the degree to which the RSSI measurement is temperature depended should be investigated in further experiments.

In order to quantify the correlation between the measurement series of the RSSI and the ion viscosity, both measurement series was normalized to values between 0 and 1 and the correlation coefficient was calculated according to Eq. 3 . The correlation coefficient of the two measurement series is 0.98 . Consequently, the two measurement series possess a very high linear correlation. Hence, the correlation coefficient confirms the optical impression that there is a very high correlation between the two measurements.

A curve fitting was performed and the functions of the two measurement series were determined to examine them in detail. The curve fitting was executed with a Gauss function, a rational function, a polynomial function, and an exponential function. The Gauss function matches the measured values of both functions with the lowest sum of squared errors
(SSE). Table 1 presents the general functions considered as well as the SSE and Table 2 details the functions with their variables.

Figure 7 visualizes the measured values, the determined gauss functions, and their first derivation. Thus, Fig. 7 displays:

- Both measurement series start after the viscosity minimum $\mathrm{tCP}(2)$ occurs.

- Both measurement series initially increase. The slope of increase of the RFID signal is higher than the increase of the DEA measurement.

- The RFID measurement series shows a turning point after approx. $50 \mathrm{~min}$. The turning point of the DEA measurement series follows at approx. $110 \mathrm{~min}$. The turning point indicates the inflection point $\mathrm{tCP}(3)$.

- The RFID measurement series levels out after approx. 400 min, but still shows a slight slope afterward. The DEA measurement series levels out after approx. $550 \mathrm{~min}$. Subsequently, the slope is almost zero. The levelling out of the slope indicates a cessation of the polymerisation reaction and, thus, the end of curing $\operatorname{tCP}(4)$. In both cases, the user can define a specific slope, which can be considered as the end of curing.

Table 1 Result of curve fitting

\begin{tabular}{|c|c|c|}
\hline \multirow[t]{2}{*}{ Function } & \multicolumn{2}{|l|}{ SSE } \\
\hline & RFID & DEA \\
\hline $\begin{array}{l}\text { Gauss } \\
f(x)=a_{1} \times \exp \left(-\left(\left(x-b_{1}\right) / c_{1}\right)^{2}\right)+a_{2} \times \exp (-(( \\
\left.\left.\left.\quad x-b_{2}\right) / c_{2}\right)^{2}\right)+a_{3} \times \exp \left(-\left(\left(x-b_{3}\right) / c_{3}\right)^{2}\right)\end{array}$ & 1.5008 & 0.0276 \\
\hline $\begin{array}{l}\text { Rational } \\
f(x)=\left(p_{1} \times x^{2}+p_{2} \times x+p_{3}\right) /\left(x^{2}+q_{1} \times x+q_{2}\right)\end{array}$ & 1.7143 & 0.5830 \\
\hline $\begin{array}{l}\text { Polynomial } \\
f(x)=p_{1} \times x^{4}+p_{2} \times x^{3}+p_{3} \times x^{2}+p_{4} \times x+p_{5}\end{array}$ & 2.9295 & 2.8432 \\
\hline $\begin{array}{l}\text { Exponential } \\
f(x)=a \times \exp (b \times x)+c \times \exp (d \times x)\end{array}$ & 1.9913 & 6.0142 \\
\hline
\end{tabular}

Fig. 6 The RSSI measurement at $865 \mathrm{MHz}$ (black asterisk) increases synchronously with the reference measurement of ion viscosity conducted with the DEA (red circle) over time (time in $\mathrm{min}$ ) (color figure online)

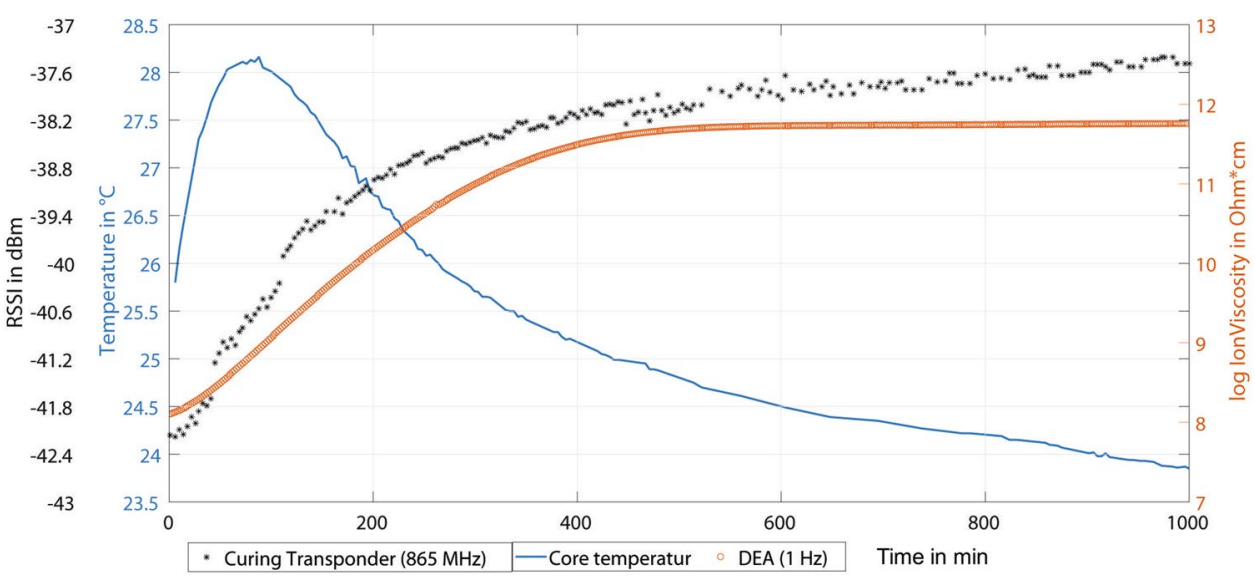


Table 2 Detailed functions

\begin{tabular}{|c|c|c|}
\hline \multirow[t]{2}{*}{ Variables } & \multicolumn{2}{|l|}{ Values } \\
\hline & RFID & DEA \\
\hline \multicolumn{3}{|l|}{ Gauss } \\
\hline$a_{1}$ & -2.24 & 11.78 \\
\hline$b_{1}$ & -38.49 & 1623.00 \\
\hline$c_{1}$ & 137.50 & $1.623 e+04$ \\
\hline$a_{2}$ & $-6.12 e+12$ & 0.06 \\
\hline$b_{2}$ & $1.426 e+04$ & 452.50 \\
\hline$c_{2}$ & 2672.00 & 137.40 \\
\hline$a_{3}$ & -37.69 & -4.01 \\
\hline$b_{3}$ & 85.08 & -98.69 \\
\hline$c_{3}$ & $1.14 \mathrm{e}+04$ & 303.60 \\
\hline \multicolumn{3}{|l|}{ Rational } \\
\hline$p_{1}$ & -37.01 & 11.32 \\
\hline$p_{2}$ & -125.70 & 38.13 \\
\hline$p_{3}$ & -107.20 & 37.03 \\
\hline$q_{1}$ & 3.36 & 3.20 \\
\hline$q_{2}$ & 2.83 & 3.15 \\
\hline \multicolumn{3}{|c|}{ Polynomial } \\
\hline$p_{1}$ & 0.2603 & -0.0434 \\
\hline$p_{2}$ & 0.5814 & 0.3358 \\
\hline$p_{3}$ & -0.1566 & -0.5367 \\
\hline$p_{4}$ & 0.1823 & 0.1836 \\
\hline$p_{5}$ & -37.9100 & 11.7800 \\
\hline \multicolumn{3}{|c|}{ Exponential } \\
\hline$a$ & -4.377 & 12.730 \\
\hline$b$ & $-7.696 e-03$ & $-6.915 e-05$ \\
\hline$c$ & -38.250 & -5.071 \\
\hline$d$ & $-1.998 \mathrm{e}-05$ & $-3.963 e-03$ \\
\hline
\end{tabular}

A detailed comparison of the two measurement series illustrates their similarity. Both measurement series follow the typical course of the DEA (displayed in Fig. 1). Nevertheless, there are differences in detail. The turning points $\mathrm{tCP}(3)$ are about $60 \mathrm{~min}$ apart. The decrease in slope and the associated end of curing $\mathrm{tCP}(4)$ can also be interpreted differently.

The differences may be due to the different positions of the sensors. Since the material under test was produced in a hand laminate process, the amount of resin remaining at the sensor positions may vary. In addition, self-heating of the resin during curing can cause the resin in the center of the laminate (position of the RFID transponder) to cure slightly faster than at the edges (position of the DEA sensor).

The result confirms the previous results of Veigt et al. [26] and demonstrates the feasibility of the UHF RFID technology for cure monitoring. The low-interference environment inside the measuring chamber leads to an almost ideal measurement curve. This result emphasizes the suitability of the UHF RFID transponder for cure monitoring. The difference of the RSSI measurement to the DEA measurement can be explained with the different position of the DEA sensor and the RFID transponder. Experiments with two DEA sensors prove that these measurement curves can also be shifted slightly to each other [26]. Questions which remain are: To which degree is the RSSI measurement temperature depended, to which degree does the DEA sensor cause interferences and how interferences can be filtered out. The filtering is essential to increase the precision and the reliable of the RSSI measurement in an environment with interference as it was the case in the preliminary work by Veigt et al.
Fig. 7 Comparison of RSSI measurement with DEA measurement
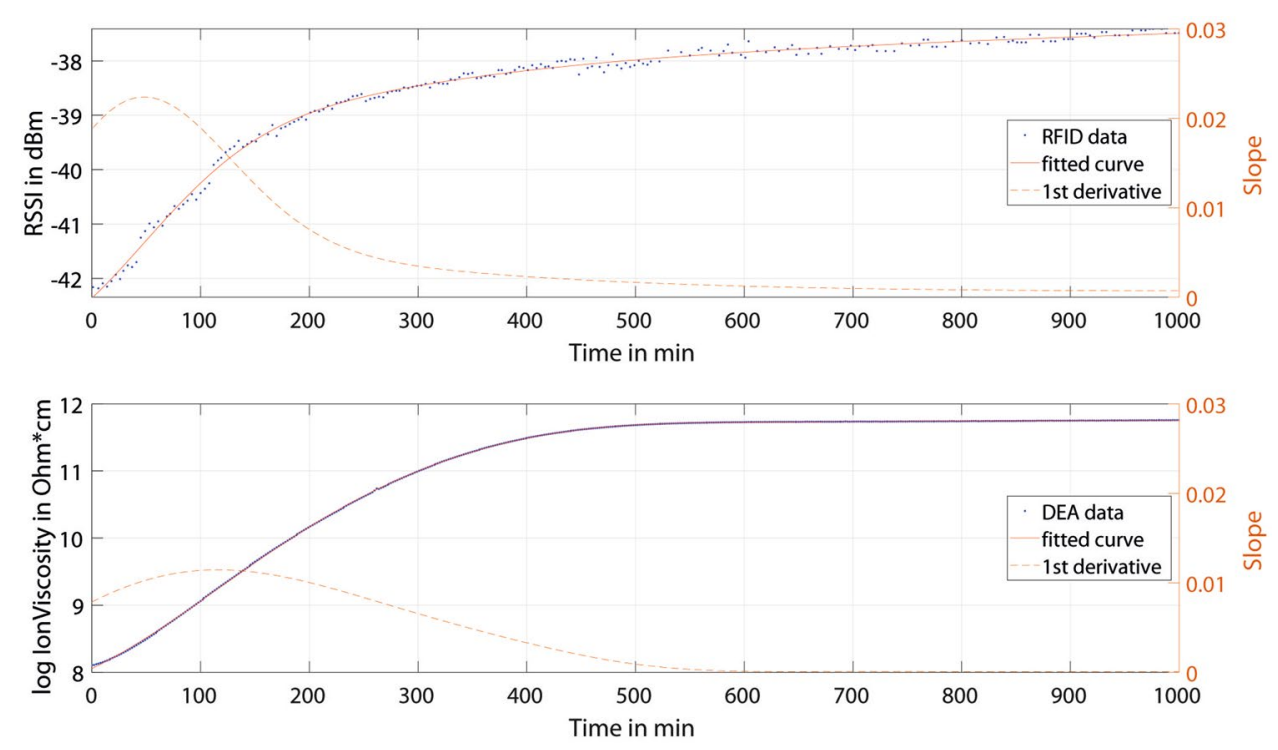


\section{Conclusion}

This contribution introduces an experimental study investigating the possibility of using RFID for curing monitoring of GFRP. The contribution considers two methods.

The frequency variation revealed that there was no shift of the high point of the response signal strength. Consequently, the optimum frequency remains the same during curing or the used transponder is not suitable for this measurement option.

At a fixed frequency, the RSSI increases over the curing time. During the experiment, all parameters was kept constant except the dielectric properties of the resin used in the GFRP. The permittivity of the transmission medium "GFRP laminate" changed over the curing time. This change influences the performance of the RFID system and leads to the increase of the RSSI. The curve of the RSSI measurement correlates very highly with the reference measurement of the DEA (correlation coefficient of 0.98). Consequently, the RSSI measurement is an indicator of the curing process of glass fiber reinforced plastics in a low-interference environment.

An advantage of using RFID is the wireless measurement. Hence, in the case of a vacuum infusion, there are no weak points in the vacuum structure due to cables. Furthermore, the transponders cost less than conventional sensors for cure monitoring, e.g. for the DEA. Compared to most conventional methods of cure monitoring, RFID has the advantage that transponders can be used in the further life of the product, e.g. for production planning and control, in logistics or maintenance. Due to these reasons, the use of RFID for cure monitoring in the manufacturing of composites can make the production more efficient. This efficiency is important in order to support the turnaround in energy policy as well as the stopping of global warming.

Further research is required to enhance the precision and reliability of the RSSI measurement in environments with interferences. For this purpose, approaches should be explored to filter out the interferences when measuring the RSSI. Besides, the suitability of the phase shift of the RFID signal should be investigated as an alternative or supplementary method and the temperature dependency of the RSSI measurement should be researched.

Acknowledgements Open Access funding provided by Projekt DEAL. This research is part of the project "Curing Transponder-Development and integration of RFID transponders to monitor curing during the manufacturing process and for product life cycle management of fiber composite components", funded by the German Federal Ministry for Economic Affairs and Energy (BMWi) under the reference numbers $16 \mathrm{KN} 021254$ and 16KN021255.

Open Access This article is licensed under a Creative Commons Attribution 4.0 International License, which permits use, sharing, adaptation, distribution and reproduction in any medium or format, as long as you give appropriate credit to the original author(s) and the source, provide a link to the Creative Commons licence, and indicate if changes were made. The images or other third party material in this article are included in the article's Creative Commons licence, unless indicated otherwise in a credit line to the material. If material is not included in the article's Creative Commons licence and your intended use is not permitted by statutory regulation or exceeds the permitted use, you will need to obtain permission directly from the copyright holder. To view a copy of this licence, visit http://creativecommons .org/licenses/by/4.0/.

\section{References}

1. Witten E, Schuster A (2019) Der Composites-Markt Europa: Marktentwicklungen, Herausforderungen und Chancen

2. Hohmann A, Albrecht S, Lindner JP et al (2018) Resource efficiency and environmental impact of fiber reinforced plastic processing technologies. Prod Eng Res Dev 12(3-4):405-417. https ://doi.org/10.1007/s11740-018-0802-7

3. Mulligan DR (2003) Cure monitoring for composites and adhesives. Rapra review reports, no. 8, vol 14. Rapra Technology Ltd, Shawbury

4. Yang Y, Chiesura G, Luyckx G et al (2014) Development of a dielectric sensor system for the on-line cure monitoring of composites. Proced Technol 15:631-637. https://doi.org/10.1016/j. protcy.2014.09.024

5. Javdanitehran M, Berg DC, Duemichen E et al (2016) An iterative approach for isothermal curing kinetics modelling of an epoxy resin system. Thermochim Acta 623:72-79. https://doi. org/10.1016/j.tca.2015.11.014

6. Kister G, Dossi E (2015) Cure monitoring of CFRP composites by dynamic mechanical analyser. Polym Test 47:71-78. https:// doi.org/10.1016/j.polymertesting.2015.08.009

7. Mizukami K, Yoshimoto S, Ogi K (2018) In-process acquisition of cure-dependent viscoelastic properties of carbon fiber reinforced composites using micromechanics-based guided wave analysis. Polym Test 65:459-467. https://doi.org/10.1016/j.polymertes ting.2017.12.032

8. Ghodhbani N, Maréchal P, Duflo H (2016) Ultrasound monitoring of the cure kinetics of an epoxy resin: identification, frequency and temperature dependence. Polym Test 56:156-166. https://doi. org/10.1016/j.polymertesting.2016.10.009

9. Schubel PJ, Crossley RJ, Boateng EKG et al (2013) Review of structural health and cure monitoring techniques for large wind turbine blades. Renew Energy 51:113-123. https://doi. org/10.1016/j.renene.2012.08.072

10. Konstantopoulos S, Fauster E, Schledjewski R (2014) Monitoring the production of FRP composites: a review of in-line sensing methods. Express Polym Lett 8(11):823-840. https://doi. org/10.3144/expresspolymlett.2014.84

11. Ding G, Cao H, Xie C (2019) Multipoint cure monitoring of temperature and strain of carbon fibre-reinforced plastic shafts using fibre Bragg grating sensors. Nondestruct Test Eval 34(2):117-134. https://doi.org/10.1080/10589759.2019.1576174

12. Kahali Moghaddam M, Breede A, Chaloupka A et al (2016) Design, fabrication and embedding of microscale interdigital sensors for real-time cure monitoring during composite manufacturing. Sens Actuators 243:123-133. https://doi.org/10.1016/j. sna.2016.03.017

13. Groh J, Lipka M, Schür J et al (2017) Wireless chipless cure monitoring sensor for fibre-reinforced plastics. In: IEEE MTT-S international microwave symposium (IMS), pp 1026-1029 
14. Composites Germany (2017) Positionen zum industriepolitischen Leichtbau-Dialog: Deutschland muss Leitanbieter werden. https ://www.composites-germany.org/images/press-releases/Positionsp apier_Leichtbau_20171120.pdf. Accessed 21 Feb 2018

15. Philipp TR, Reinhart G (2012) Evaluation of RFID technology application in production of fiber-reinforced plastics. In: ElMaraghy HA (ed) Enabling manufacturing competitiveness and economic sustainability: Proceedings of the 4th international conference on changeable, agile, reconfigurable and virtual production (CARV2011), Montreal, Canada, 2-5 October 2011. Springer, Berlin, Heidelberg, pp 263-268

16. Philipp TR, Schreiber M, Reinhart G (2013) Vorausschauende Steuerung der Auftauprozesse für Prepreg-Materialien. wt-online 103(4):317-323

17. Veigt M, Ganji F, Morales Kluge E et al. (2011) Autonomous control in production planning and control: how to integrate autonomous control into existing production planning and control structures. In: Autonomous cooperation and control in logistics: contributions and limitations : theoretical and practical perspectives. Springer, Heidelberg, pp 313-329

18. Reinhart G, Philipp T, Ostgathe M (2010) Einsatz von RFID bei der Herstellung von Faserverbundwerkstoffen. ZWF 105(09):785790. https://doi.org/10.3139/104.110395

19. Zettler J, Philipp TR, Reinhart G et al (2008) Influence of integration RFID transponders on structural behavior of carbon-fibrereinforced plastics. In: Fabozzi FJ, Kothari V (eds) Introduction to securitization. Wiley, Hoboken, pp 1-12

20. Gray B (2015) Embedding integrated RFID into fibre reinforced plastics during the manufacturing process, PhD Dissertation, University of Texas at Arlington

21. Bernhard J, Dräger T, Grabowski C et al (2011) Integration RFID in Fibre-Reinforce Plastics. In: 7th European workshop on smart object systems: technologies and applications, vol 229, Dresden, Germany

22. Reinhart G, Philipp TR, Bernhard J et al (2012) Integrating RFID transponders into fibre-reinforced plastics by using textile production technology. In: Smart SysTech 2012: European conference on smart objects, systems and technologies, pp 1-6
23. Hardi E, Veigt M, Koerdt M et al (2018) Use of RFID tags for monitoring resin flow and investigation of their influence on the mechanical properties of the composite. Procedia Manuf 24:305310. https://doi.org/10.1016/j.promfg.2018.06.023

24. Veigt M, Hardi E, Koerdt M et al (2018) RFID-Curing-Transponder RFID als ein neuer Ansatz zur Aushärtungsüberwachung von Komponenten aus glasfaserverstärktem Kunststoff. Ind Manag 34(4):7-10

25. Veigt M, Cen M, Hardi E, Lang W, Freitag M (2020) Using RFID to monitor the curing of aramid fiber reinforced polymers. In: Freitag M, Haasis H-D, Kotzab H, Pannek J (eds) Dynamics in logistics. Proceedings of the 7 th international conference on dynamics in logistics (LDIC 2020). Springer, Cham, pp 441-450

26. Veigt M, Hardi E, Koerdt M et al (2018) Curing transponderintegrating RFID transponder into glass fiber-reinforced composites to monitor the curing of the component. Procedia Manuf 24:94-99. https://doi.org/10.1016/j.promfg.2018.06.014

27. Groh J, Lee WB, Schur J et al. (2018) Compact substrate integrated wireless cure monitoring sensor for epoxy resin. In: 2018 48th European microwave conference (EuMC). IEEE, pp 243-246

28. Finkenzeller K (2015) RFID-Handbuch: Grundlagen und praktische Anwendungen von Transpondern, kontaktlosen Chipkarten und NFC, 7, aktualisierte und, erweiterte edn. Hanser, München

29. Lee HL (2014) The handbook of dielectric analysis and cure monitoring. Lambient Technologies, Cambridge

30. Mijovic J, Yee CFW (1994) Use of complex impedance to monitor the progress of reactions in epoxy/amine model systems. Macromolecules 27(25):7287-7293. https://doi.org/10.1021/ma001 $03 \mathrm{a} 009$

31. Fahrmeir L, Heumann C, Künstler R et al (2016) Statistik. Springer, Berlin

Publisher's Note Springer Nature remains neutral with regard to jurisdictional claims in published maps and institutional affiliations. 